\title{
Mathematical Morphology in the HLS Colour Space
}

\author{
Allan Hanbury and Jean Serra
}

\begin{abstract}
The HLS colour space is widely used in image analysis as it is physically intuitive. As the hue component of this space is defined on the unit circle, standard greyscale image analysis operators, specifically morphological operators, are not applicable to it. A variation of the standard morphological operators which require the choice of an origin are discussed. In addition, some lexicographical vector orders, which allow mathematical morphology to be used in the HLS space, are presented. Included in these is a new saturationweighted hue order, which takes hue and saturation into account simultaneously.
\end{abstract}

\section{Introduction}

Many colour spaces attempt to separate luminance and chrominance information. Colours specified in the coordinates of these spaces are thought to correspond more closely to human colour intuition than spaces such as RGB or CMYK. In general, one vector component is used to specify the luminance, and two to specify the chrominance. These two are usually an angle to express the hue (colour), and a fraction to express the colour purity.

A variety of such colour spaces are commonly used. One of the most widespread of the separate luminance and chrominance spaces is the HLS space and its relatives (HSI, HSV, etc.). Even though this colour space has a number of disadvantages [8], the techniques introduced in this article are applied to this space due to its ubiquity.

The use of many image analysis operators on colour images is not evident. For the particular case of the morphological operators, one is faced with the problem of imposing an order on vectorial data. In addition, in a colour space where one of the components is represented by a variable on the unit circle (the hue), further problems present themselves. A number of possible orders have been suggested for vectors in the RGB space [1, 2, 3, 9], and methods of applying mathematical morphology to data on the unit circle have been proposed [5]. In this article, some of these techniques are modified to operate on the HLS colour space in a physically intuitive way.

Given that the hue is defined on the unit circle (i.e. for any angle $\theta, \theta=\theta+2 \pi n, n \in$ $\mathbb{Z}$ ), we cannot apply standard greyscale techniques to its treatment. Some operators adapted to the treatment of circular data are therefore presented. Section 2 discusses the ordering of hues requiring the choice of an origin. When one processes colour images, it is preferable not to treat each component separately, as recombining the components after processing can lead to the introduction of false colours. Section 3 therefore discusses some lexicographical vector orders in the HLS space, including a new method of using the saturation values to weight the hue values. Section 4 concludes. 


\section{Ordering the hue component}

As the hue is defined on the unit circle, the values wrap around $(\theta=\theta+2 \pi n, n \in \mathbb{Z})$, and we cannot directly build a lattice for the hue values. Some rotationally invariant operators on the unit circle have been defined [5], and can be applied to the hue band, but they have not yet been expanded to the full HLS space.

A method for ordering the hue values which requires the choice of an origin has been proposed by Peters [7]. Consider some arbitrary points $a_{i}$ distributed on the unit circle with centre $o$. After choosing an origin $a_{0}$, the distance from each point $a_{i}$ to the origin is defined as the acute angle $\widehat{a_{i} O a_{0}}$, or

$$
D_{i}=D\left(a_{i}, a_{0}\right)=a_{i} \div a_{0}=\left\{\begin{array}{lll}
\left|a_{i}-a_{0}\right| & \text { if } & \left|a_{i}-a_{0}\right| \leq 180^{\circ} \\
360^{\circ}-\left|a_{i}-a_{0}\right| & \text { if } & \left|a_{i}-a_{0}\right| \geq 180^{\circ}
\end{array}\right.
$$

The Peters algorithm for mathematical morphology on a hue image is to choose a global hue origin $H_{0}$, and to calculate the distance $D_{i}$ for all the points in the structuring element. The point with smallest $D_{i}$ is considered to be the infimum of the structuring element, and the point with the largest $D_{i}$ to be the supremum. The erosion is thus the point in the structuring element closest to the origin, and the dilation the point furthest from the origin.

This definition is, however, intuitively upside-down. Consider the simple two-colour example in Figure 1a, an image in the HLS space having grains made up of red pixels with coordinates $(H, L, S)=\left(0^{\circ}, \frac{1}{2}, 1\right)$ and the background consisting of yellow pixels with coordinates $\left(60^{\circ}, \frac{1}{2}, 1\right)$. If one chooses $H_{0}=0^{\circ}$ (i.e. the hue of the grains) and performs a dilation using the Peters formulation, one actually erodes the grains and dilates the pores (Figure 1b). In order to allow the user to choose the origin as having the colour of the objects of interest, the Peters definition is inverted, and the hue erosion and dilation are defined as

$$
\varepsilon_{H} H(\mathbf{x})=\left\{H(\mathbf{y}): D\left(H(\mathbf{y}), H_{0}\right)=\sup \left[D\left(H(\mathbf{z}), H_{0}\right)\right], \mathbf{z} \in B_{\mathbf{x}}\right\}
$$

and

$$
\delta_{H} H(\mathbf{x})=\left\{H(\mathbf{y}): D\left(H(\mathbf{y}), H_{0}\right)=\inf \left[D\left(H(\mathbf{z}), H_{0}\right)\right], \mathbf{z} \in B_{\mathbf{x}}\right\}
$$

where $H(\mathbf{x})$ is the value of the hue at point $\mathbf{x}$ in an image, $B_{\mathbf{x}}$ indicates an arbitrary structuring element $B$ at point $\mathbf{x}$, and the value of $H_{0}$ is chosen in advance. The result of a dilation using equation 3 is shown in Figure 1c.

It is clear that a hue order based only on equation 1 is a pre-order. The points $a_{1}=a_{0}+\left(\theta \div a_{0}\right)$ and $a_{2}=a_{0}-\left(\theta \div a_{0}\right)$, where $\theta$ is an arbitrary angle, have the same distances from the origin, even though they are not the same point. We can impose a complete order by stating, for example, that in the situation where $D\left(a_{1}, a_{0}\right)=D\left(a_{2}, a_{0}\right)$ and $a_{1} \neq a_{2}$, we take $a_{1}>a_{2}$ if $\left(a_{1} \bmod 360^{\circ}\right)<180^{\circ}$, else $a_{1}<a_{2}$.

\section{Vector orders in the HLS space}

The ranges of the components in the HLS space are taken to be $0^{\circ} \leq H \leq 360^{\circ}, 0 \leq$ $L \leq 1$ and $0 \leq S \leq 1$, but the algorithms presented can easily be adapted to other ranges. 


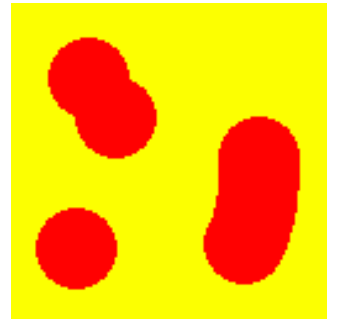

(a)

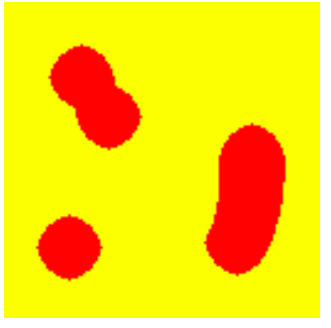

(b)

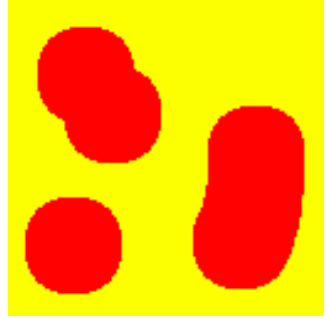

(c)

Figure 1: (a) The original "binary colour" image containing red grains and yellow pores (background); (b) The dilation of image (a) by the Peters formulation; (c) the dilation of image (a) using equation 3 . The hue origin $H_{0}=0^{\circ}$ was used for both operations.

Before applying any vector orders to colours in the HLS space, one should ensure that the double-cone saturation values are being used. Because of the double-cone shape of the HLS space, the maximum possible saturation value depends on the luminance value. For example, at the luminance axis extremities $L=0$ and $L=1$, the maximum saturation value is 0 . The saturation can only take on its full range of values at $L=\frac{1}{2}$. Many RGB to HLS transformations available in image processing packages produce an image in a cylindrical version of the HLS space, in which the saturation values encode the fraction of the maximum possible saturation value for the corresponding luminance. This implies that the saturation can take on the full range of values for every colour, but that ordering the colours based on this representation is meaningless [4].

In order to use mathematical morphology operations in a vector space, such as the HLS space, we need to order the vectors so as to avoid introducing false colours. This enables one to choose a supremum or infimum from amongst the vectors (not vector components) of a set. If one splits an HLS image into its three channels, dilates only the saturation channel, and then recombines the three channels, false colours (i.e. colours which were not present in the initial image) will often be introduced.

The HLS space permits one to apply some physically intuitive orders to the vectors, and some possible lexicographical orders for the vectors are presented. The standard lexicographical order for arbitrary three-component vectors is defined as follows:

$$
\left(a_{1}, b_{1}, c_{1}\right)<\left(a_{2}, b_{2}, c_{2}\right) \quad \text { if }\left\{\begin{array}{l}
a_{1}<a_{2} \\
\text { or } \\
a_{1}=a_{2} \text { and } b_{1}<b_{2} \\
\text { or } \\
a_{1}=a_{2} \text { and } b_{1}=b_{2} \text { and } c_{1}<c_{2}
\end{array}\right.
$$

The lexicographical order is a complete order (and is the order in which words are arranged in dictionaries, hence the name). In the RGB space, one can apply relation 4 directly to the vectors, although this implies having to elevate one of the $R, G$ or $B$ components to prominence, a choice which is not always obvious. Due to the specific shape of the HLS space and the angular hue component, the straight application of relation 4 to this space is not possible. Possible formulations are discussed in this section. Firstly, 


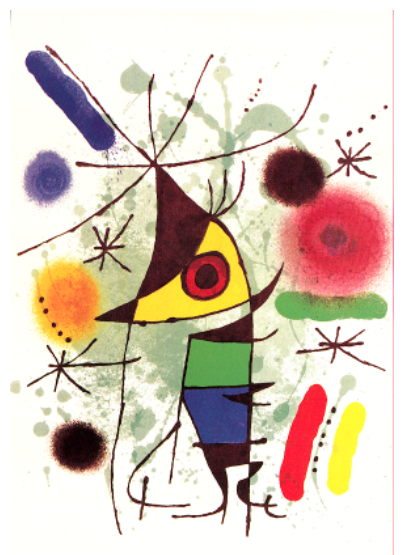

Figure 2: "Le chanteur" by Joan Mirò

orders having luminance or saturation at the first level, and then the more difficult task of the lexicographical order with the hue component at the first level.

The examples in this section use an image of the painting "Le chanteur" by Joan Mirò, and the original colour image is reproduced in Figure 2 for comparative purposes.

\subsection{Vector erosion and dilation}

Once a vector order has been defined, thereby allowing the choice of a supremum and infimum from a set of vectors, one can define the basic morphological operators. The vector erosion of an image $f: \mathbb{R}^{2} \rightarrow \mathbb{R}^{3}$ at point $\mathbf{x}$ by structuring element $B$ is

$$
\varepsilon f(\mathbf{x})=\left\{f(\mathbf{y}): f(\mathbf{y})=\inf [f(\mathbf{z})], \mathbf{z} \in B_{\mathbf{x}}\right\}
$$

and the corresponding dilation by structuring element $B$ is

$$
\delta f(\mathbf{x})=\left\{f(\mathbf{y}): f(\mathbf{y})=\sup [f(\mathbf{z})], \mathbf{z} \in B_{\mathbf{x}}\right\}
$$

\subsection{Luminance and saturation}

Choosing to order the vectors by luminance or saturation alone is straightforward, as these components form complete lattices. Using them as the first level in a lexicographical order is therefore obvious.

For the HLS vectors $\mathbf{c}_{i}$, the lexicographical order with luminance in the first position is defined as:

$$
\mathbf{c}_{i}>\mathbf{c}_{j} \text { if }\left\{\begin{array}{l}
L_{i}>L_{j} \\
\text { or } \\
L_{i}=L_{j} \text { and } S_{i}<S_{j} \\
\text { or } \\
L_{i}=L_{j} \text { and } S_{i}=S_{j} \text { and } H_{i} \div H_{0}<H_{j} \div H_{0}
\end{array}\right.
$$

The orders for the saturation and hue in the second and third rows are somewhat arbitrary, and the orders of the signs could easily be reversed. For the third row, one 


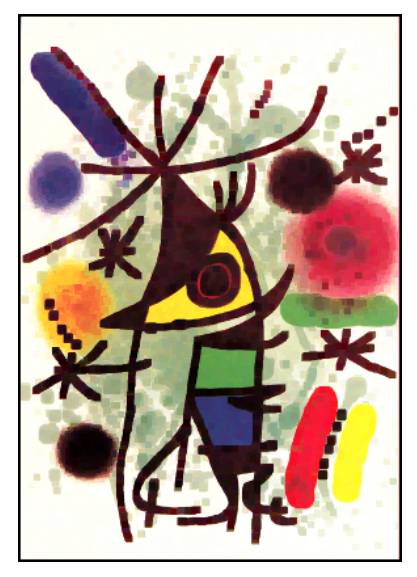

(a) Erosion

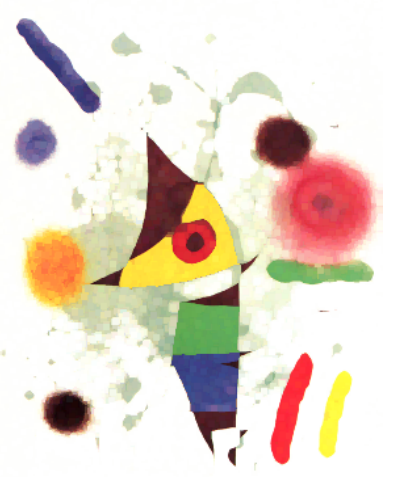

(b) Dilation

Figure 3: The luminance erosion and dilation with a square of size $2(5 \times 5$ pixels $)$.

has to choose an arbitrary hue origin $H_{0}$, but in practice this parameter generally has a negligible effect on the results, as the "decision process" almost never reaches the third row. An example of the use of this lexicographical order with the erosion and dilation operators is shown in Figure 3.

The lexicographical order with saturation in first position is defined as

$\mathbf{c}_{i}>\mathbf{c}_{j}$ if $\left\{\begin{array}{l}S_{i}>S_{j} \\ \text { or } \\ S_{i}=S_{j} \quad \text { and } \quad\left|L_{i}-0.5\right|<\left|L_{j}-0.5\right| \\ \text { or } \\ S_{i}=S_{j} \quad \text { and } \quad\left|L_{i}-0.5\right|=\left|L_{j}-0.5\right| \quad \text { and } \quad H_{i} \div H_{0}<H_{j} \div H_{0}\end{array}\right.$

The saturation dilation chooses the pixel in the structuring element with the "purest" or most saturated colour. For a saturation dilation, where two or more pixels in a structuring element have saturation values equal to the supremum, the one with luminance component closest to $L=\frac{1}{2}$ is chosen, as at this luminance value the saturation can assume its largest range of values. Examples of saturation morphological operations are shown in Figure 4.

\subsection{Hue}

\subsubsection{Hue only}

For the hue band, the most obvious approach is to simply expand the hue morphology described in section 2 and to construct a lexicographical order with, in first position, the hue order based on distance from a chosen hue (hue origin), followed by saturation and luminance in second and third positions. However, because the hue and saturation components interact closely, this ordering leads to poor results. 


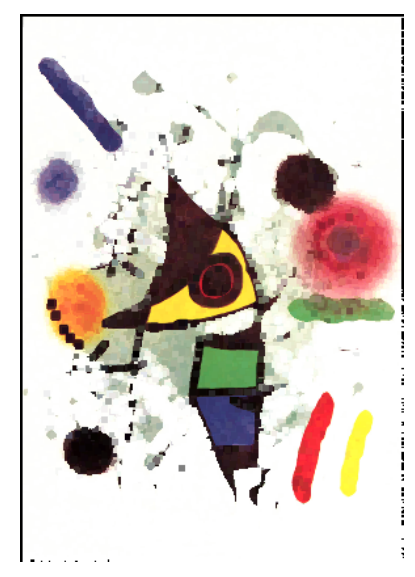

(a) Erosion

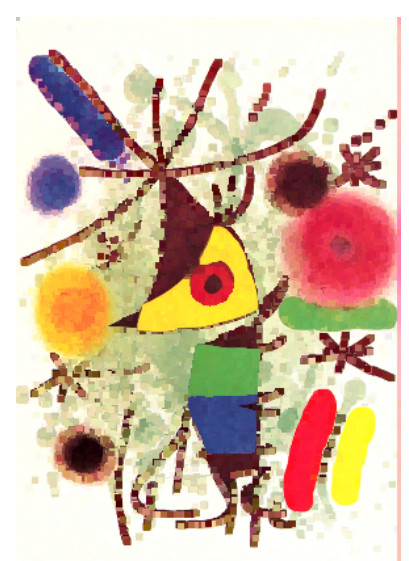

(b) Dilation

Figure 4: The saturation erosion and dilation with a square of size $2(5 \times 5$ pixels $)$.

The results of using this simple order with $H_{0}=30^{\circ}$ (corresponding to the fully saturated colour ) are shown in Figure $6 \mathrm{a}$ for erosion and $6 \mathrm{c}$ for dilation. With this choice of hue origin, a dilation should enlarge the reds and yellows, and shrink the blues and purples. It is clear, for example in the dilation of the red stripe at the bottom right of the image (Figure 6c), that white pixels are often chosen in preference to the red pixels, even though, intuitively, we expect the red pixels to have preference. This is because some of the red pixels have hue values around $350^{\circ}$, and the surrounding white pixels have hue values around $0^{\circ}$, which are closer to $H_{0}=30^{\circ}$ using the angular hue distance criterion, and these are therefore chosen.

The drawback with ordering only by hue is further illustrated in Figure 5. If one chooses the colour closest to red $a=\left(0^{\circ}, 0.5,1.0\right)$ in this image using only the hue distance, the result is the brown with coordinates $c=\left(10^{\circ}, 0.5,0.2\right)$, although the orange $b=\left(20^{\circ}, 0.5,0.9\right)$ is visually the most similar. A possible solution to this problem, which weights hue values by their corresponding saturations before ordering them, is proposed.

\subsubsection{Saturation-weighted hue}

The proposed solution adds a preprocessing step in which a new saturation-weighted hue value $H^{\prime}$ is associated with each vector, and these new hue values are used when assigning an order based on the distance from the selected origin. The principal characteristics of the saturation-weighted hue $H^{\prime}$ are:

- Vectors with a high saturation value keep their initial hue value.

- Vectors with a low saturation value are moved towards the $H_{0}+90^{\circ}$ or $H_{0}-90^{\circ}$ positions to reduce the probability of being chosen as the supremum or infimum.

Once the sup or inf has been chosen, the vectors are reassigned their initial hue value $H$ to avoid introducing false colours. 


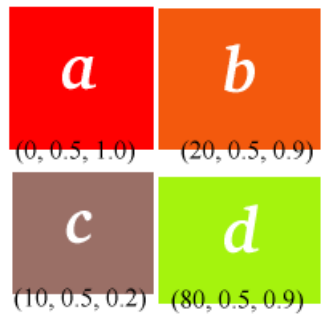

(a)

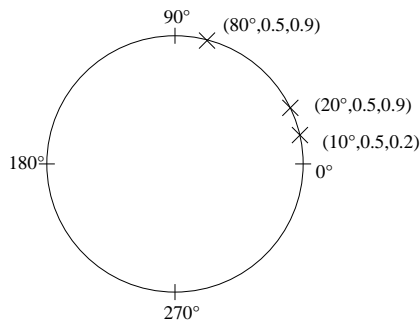

(b)

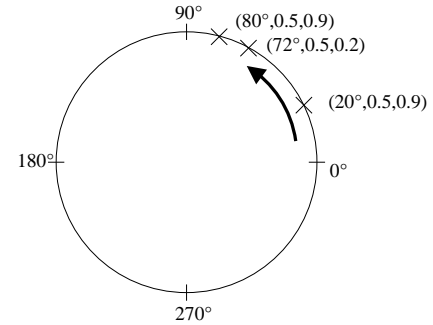

(c)

Figure 5: (a) Four colours and their HLS values, which illustrate the problem of using only the hue value to order the colours; (b) The position of these colours on the hue circle using values $H_{i}$; (c) The position of the colours using values $H_{i}^{\prime}$.

Before giving a general formulation, we will treat the specific example in Figure 5. We choose the origin $H_{0}=0^{\circ}$, and calculate $H^{\prime}$ as described here. The positions on the hue circle of the three colours (excluding colour $a$ ) in Figure 5a are shown in Figure 5b. All three points are between $0^{\circ}$ and $90^{\circ}$, so we assign

$$
H_{i}^{\prime}=\sup \left[H_{i}, 90^{\circ}\left(1-S_{i}\right)\right]
$$

Consider point $c=\left(10^{\circ}, 0.5,0.2\right)$ which has the hue value $H_{c}=10^{\circ}$ closest to the origin, and for which $90^{\circ}-\left(0.2 \times 90^{\circ}\right)=72^{\circ}$. We therefore, using equation 9 , set $H_{c}^{\prime}=72^{\circ}$. For the points $b=\left(20^{\circ}, 0.5,0.9\right)$ and $d=\left(80^{\circ}, 0.5,0.9\right)$, the expression $90^{\circ}-\left(0.9 \times 90^{\circ}\right)=9^{\circ}$, so $H_{b}^{\prime}=H_{b}$ and $H_{d}^{\prime}=H_{d}$. The positions of the points on the hue circle using the values of $H^{\prime}$ are shown in Figure 5c. In the subsequent dilation using ordering by the values of $H^{\prime}$, we would pick the point $b$ as the closest to the origin.

The general formulation of the preprocessing, which takes the four quadrants of the circle into account, is given below. For each point $i$, a value $H_{i}^{\prime}$ is calculated. In order to simplify the notation, it is assumed that the origin $H_{0}$ has the value of $0^{\circ}$. To take this into account in practice, one simply performs the following substitution

$$
H_{i} \rightarrow\left\{\begin{array}{lll}
H_{i}-H_{0} & \text { if } \quad H_{i}-H_{0} \geq 0 \\
360^{\circ}+\left(H_{i}-H_{0}\right) & \text { if } & H_{i}-H_{0}<0
\end{array}\right.
$$

in the equation for calculating $H^{\prime}$, which is

$$
H_{i}^{\prime}=\left\{\begin{array}{lll}
\sup \left[H_{i}, 90^{\circ}\left(1-S_{i}\right)\right] & \text { if } & 0^{\circ} \leq H_{i} \leq 90^{\circ} \\
\inf \left[H_{i}, 90^{\circ}\left(1+S_{i}\right)\right] & \text { if } & 90^{\circ}<H_{i} \leq 180^{\circ} \\
\sup \left[H_{i}, 90^{\circ}\left(3-S_{i}\right)\right] & \text { if } & 180^{\circ}<H_{i} \leq 270^{\circ} \\
\inf \left[H_{i}, 90^{\circ}\left(3+S_{i}\right)\right] & \text { if } & 270^{\circ}<H_{i}<360^{\circ}
\end{array}\right.
$$


The lexicographical order based on the saturation-weighted hue is defined as

$\mathbf{c}_{i}>\mathbf{c}_{j}$ if $\left\{\begin{array}{l}\left(H_{i}^{\prime} \div 0^{\circ}\right)<\left(H_{j}^{\prime} \div 0^{\circ}\right) \\ \text { or } \\ \left(H_{i}^{\prime} \div 0^{\circ}\right)=\left(H_{j}^{\prime} \div 0^{\circ}\right) \text { and }\left|L_{i}-0.5\right|<\left|L_{j}-0.5\right| \\ \left.\text { or } H_{i}^{\prime} \div 0^{\circ}\right)=\left(H_{j}^{\prime} \div 0^{\circ}\right) \text { and }\left|L_{i}-0.5\right|=\left|L_{j}-0.5\right| \text { and } S_{i}>S_{j}\end{array}\right.$

Here, once again, the directions of the comparisons for the luminance and saturation in the second and third rows are arbitrarily chosen. The difference between using the lexicographical order with hue in the first position (section 3.3.1) and the operators using the saturation-weighted hue (equation 10) is demonstrated in Figure 6 for the erosion and dilation operators. The origin $H_{0}$ is chosen to be at $30^{\circ}$, so that a dilation should enlarge the reds and yellows and shrink the blues and purples. As has already been pointed out, one can see in Figure $6 \mathrm{c}$ that this is not true when only the hue is used. The red stripe at the bottom right of the image has a hue value at around $350^{\circ}$, and the dilation should therefore result in its expansion. Even though the dilation based on ordering by hue only fails (Figure 6c) due to the surrounding white pixels having a hue of $0^{\circ}$, the saturationweighted hue dilation (Figure 6d) eliminates these pixels from consideration due to the saturation weighting.

A disadvantage of this approach is that in regions which do not contain highly saturated pixels with hue close to the selected origin, the behaviour is less predictable. This is visible, for example, in the green regions of the image in Figure 6.

\section{Conclusion}

This article deals principally with morphological operators for the HLS colour space. This colour space is widely used in image analysis because it is physically intuitive, even though it has some disadvantages of which the user should be aware. Most of the difficulties of applying image analysis operators in this space are due to the hue component being defined on the unit circle. Unneeded difficulties can be introduced through the inadvertent use of the cylindrical version of the HLS space.

A discussion and development of morphological operators requiring the choice of an origin is presented. In addition, some lexicographical orders for the HLS space are suggested. The first two orders simply use the two components which already possess an order - the luminance and saturation - in first position. The problems with a "traditional" lexicographical order with hue in first position are discussed, and a solution in the form of a lexicographical order with saturation-weighted hue in first position is suggested.

Having presented these orders, it is interesting to reflect on the practical image analysis tasks to which they are applicable, and which one is best applied to which task. The choice depends largely on the properties of the images to be processed, and the information that the user wishes to extract from these images. Some possible applications are:

- Operators based on the lexicographical order with luminance in first position are the best at preserving the contours of the objects in the image. Nevertheless, they are useful only in situations where one knows that the objects of interest are the brightest or darkest in the image. 


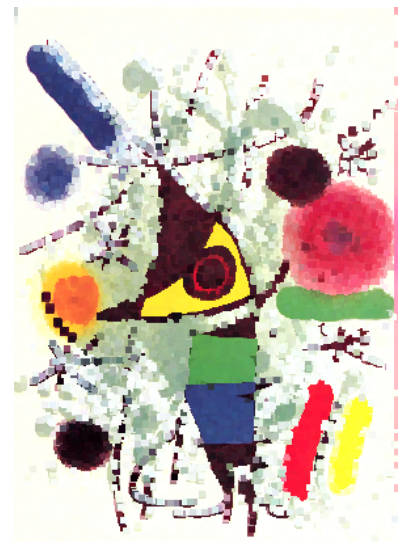

(a) Hue-only erosion

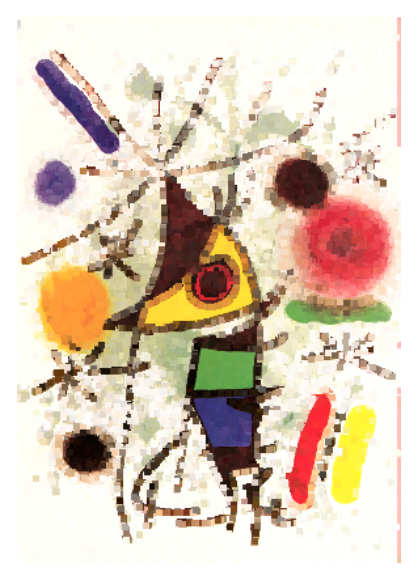

(c) Hue-only dilation

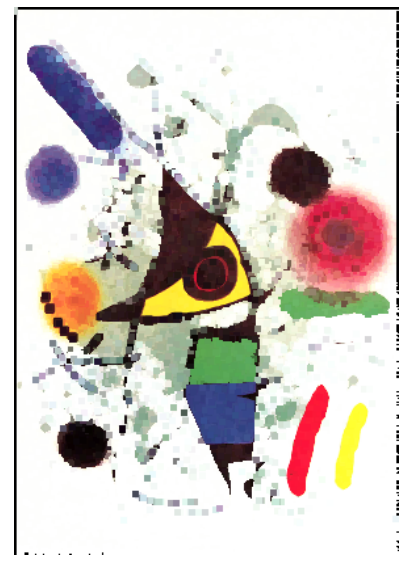

(b) Saturation-weighted hue erosion

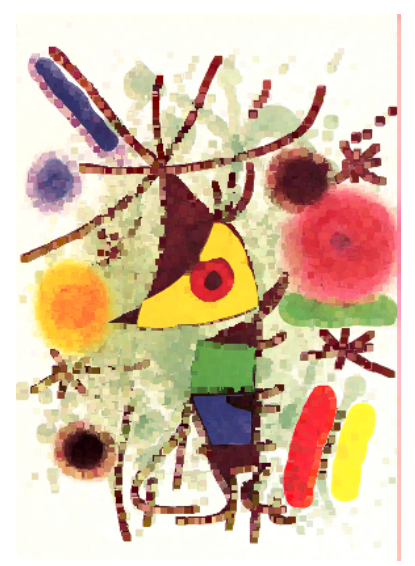

(d) Saturation-weighted hue dilation

Figure 6: The erosion and dilation with a square of size 2 and the origin $H_{0}=30^{\circ}$. Hueonly ordering is used in (a) and (c), and the proposed saturation-weighted hue ordering in (b) and (d). 
- In situations where the objects of interest are highly coloured on a greyish background, the operators with saturation in first position should be best. However, the choice between a low saturation black and a low saturation white pixel made by the operator can appear to be arbitrary. This problem also manifests itself in textured regions containing colours of different hues but similar saturations.

- In an application where only objects of a specific colour are of interest, the saturationweighted hue operators with an astutely chosen hue origin can be used to smooth these objects before thresholding, thus making the threshold range smaller. When using the saturation-weighted hue operators, it should be remembered that even though one gains a "better" behaviour around the hue origin $H_{0}$ and the point $H_{0}+180^{\circ}$, the choices made by the operator in regions of the image which contain no pixels with hues close to these points can appear arbitrary.

Even though only the HLS colour space is discussed, the techniques should be applicable to other spaces with angular hue values, such as HSI, HSV or the polar version of the L*a*b* space [6]. For these spaces, some small modifications to the orders could be necessary to take into account the different shapes of the spaces.

\section{References}

[1] J. Chanussot and P. Lambert. Total ordering based on space filling curves for multivalued morphology. In Proceedings of the International Symposium on Mathematical Morphology (ISMM '98), pages 51-58, 1998.

[2] Mary L. Comer and Edward J. Delp. Morphological operations for colour image processing. Journal of Electronic Imaging, 8(3):279-289, 1999.

[3] M.C. d'Ornellas, R. Boomgaard, and J. Geusebroek. Morphological algorithms for color images based on a generic-programming approach. In Proceedings of the XI Brazilian Symposium on Computer Graphics and Image Processing (SIBGRAPI'98). IEEE Press, 1998.

[4] Allan Hanbury. Lexicographical order in the HLS colour space. Technical Report N04/01/MM, Centre de Morphologie Mathématique, Ecole des Mines de Paris, 2001.

[5] Allan Hanbury and Jean Serra. Morphological operators on the unit circle. Accepted for publication by IEEE Transactions on Image Processing.

[6] Allan Hanbury and Jean Serra. Mathematical morphology in the $\mathrm{L}^{*} \mathrm{a} \mathrm{b}^{*}$ space. In Proceedings of the 8th European Congress for Stereology and Image Analysis, 2001.

[7] R. A. Peters II. Mathematical morphology for angle-valued images. In Non-Linear Image Processing VIII. SPIE volume 3026, 1997.

[8] Charles Poynton. Frequently asked questions about color. URL: http://www.inforamp.net//poynton/PDFs/ColorFAQ.pdf, 1999.

[9] H. Talbot, C. Evans, and R. Jones. Complete ordering and multivariate mathematical morphology: Algorithms and applications. In Proceedings of the International Symposium on Mathematical Morphology (ISMM '98), pages 27-34, 1998. 